\title{
TRIPHALANGEAL THUMB IN A BANTU FAMILY
}

\author{
I. Abramowitz, Johannesburg, South Africa
}

Surgical Registrar, Coronation Hospital and University of the Witwatersrand

Triphalangeal thumb is a rare and interesting anomaly with a strong hereditary tendency. The present communication reports its occurrence in three generations of a Bantu family. Incidence-The first report of a triphalangeal thumb was published by Struthers in 1863. and its comparative rarity may be indicated by the fact that in a review of the literature for the ensuing fifty-two years (Stieve 1916) only thirty-nine cases were collected.

Lapidus, Guidotti and Coletti (1943) discovered six cases while examining 75.000 men drafted to an American Army centre. These workers quoted a statement by Dr M. Pomeranz. radiologist of the Hospital for Joint Diseases in New York, that during the past twenty-five years there had not been seen a single case of triphalangeal thumb in his department.

During the past decade sporadic reports have appeared in both English and Continental journals. The occurrence of triphalangeal thumb in the Negro has been reported by Frere (1930). In South Africa the only previous report of its occurrence in the Bantu was made by McGregor (1926).

\section{CASE REPORTS}

Case 1-A Bantu boy (Shangaan), a first-born child aged eight years, was admitted to the Coronation Hospital in February 1958 under Mr B. Lewin, with a supracondylar fracture of the left humerus. During the examination it was noted that both his thumbs were long, the

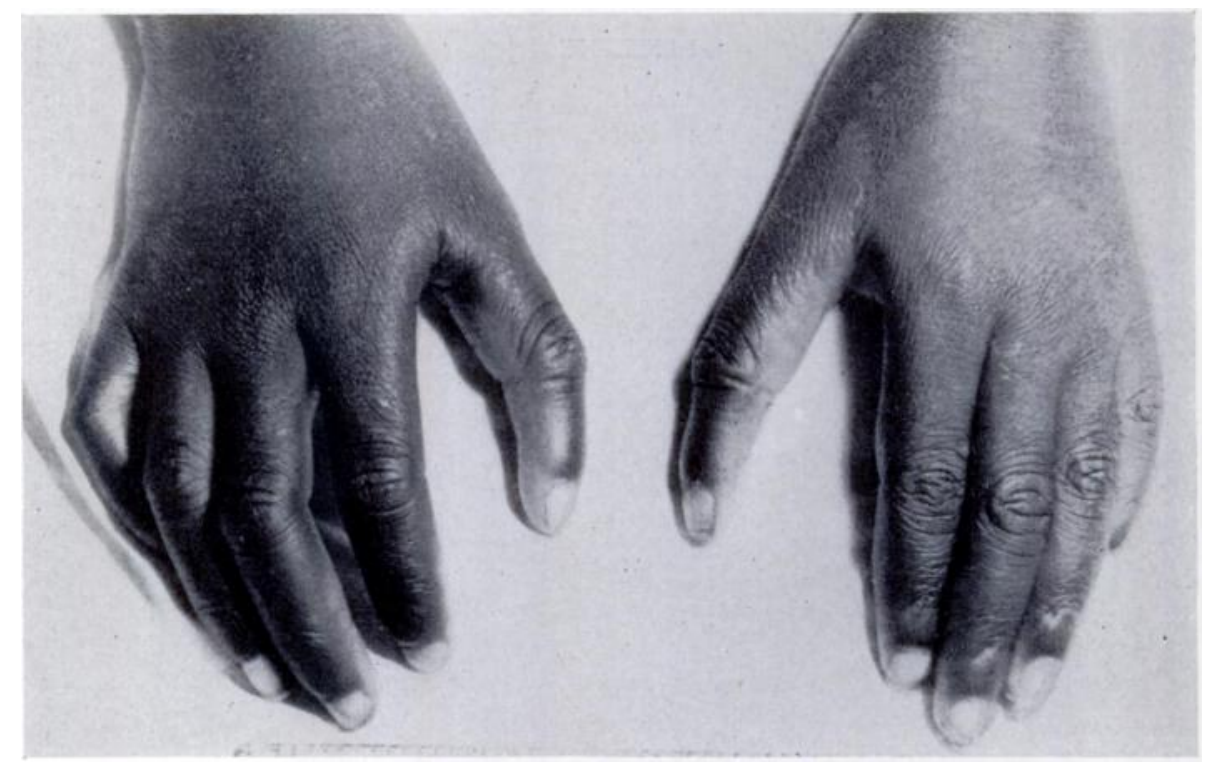

FIG. 1

Case 1

tip of each reaching to the level of the middle of the middle phalanx of the index finger (Fig. 1). Both thumbs consisted of three phalangeal elements. The distal phalanges were held in slight flexion and ulnar deviation. The skin creases overlying the dorsum of the distal interphalangeal joints were smooth, but those over the proximal interphalangeal joints were well marked. 
Movements of the distal interphalangeal joints were restricted to about 5 degrees of palmar flexion, whereas the proximal interphalangeal joints were freely mobile. The metacarpophalangeal joints were not only freely mobile in flexion and extension but also possessed an unusually wide range of abduction and adduction. The carpo-metacarpal joint movements were normal.

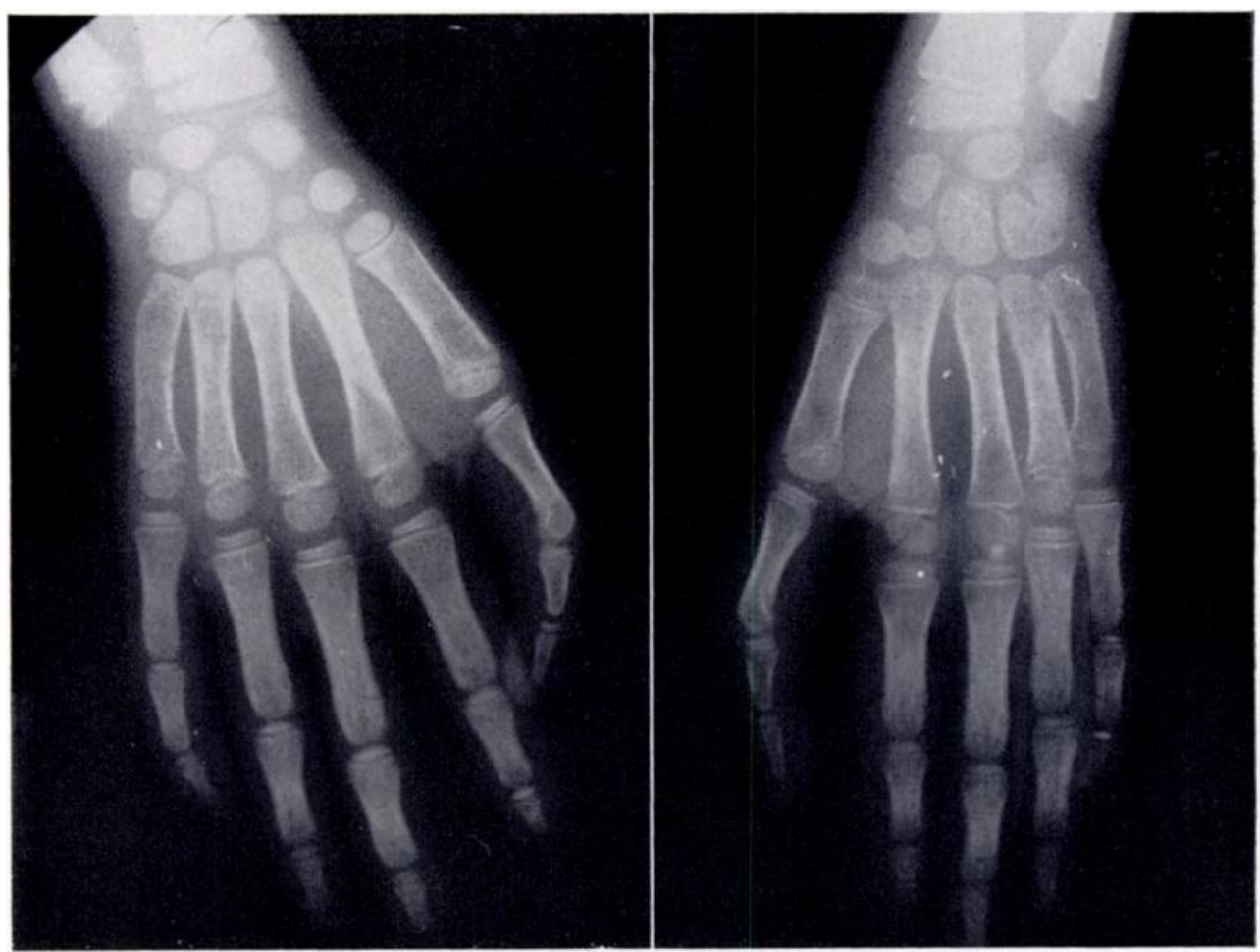

Fig. 2

Case 1

Radiographs (Fig. 2) confirmed the presence of triphalangeal thumbs: all three phalanges were well formed on both sides. Both metacarpals possessed basal and capital epiphyses. Radiological examination showed both feet to be normal. No other anomalies were noted.

Case 2-The woman in this case was a Bantu (Shangaan) aged twenty-five years, and the mother of the patient in Case 1. Like her son she was a first-born child and neither her brother nor her two sisters had an abnormality of the thumbs. She has three children, but the boy described in Case 1 alone has the anomaly. (The other two children were not examined by the author.)

Both her thumbs appeared relatively long (Fig. 3). The tip of the left thumb reached to the level of the proximal interphalangeal joint of the index finger. The right thumb was slightly longer and reached to the level of the middle of the middle phalanx of the index finger. The distal phalanges of both thumbs were held in slight flexion and ulnar deviation. The skin creases over the dorsum of both thumbs were absent. Both distal interphalangeal joints were relatively immobile, movement being restricted to about 5 degrees of flexion. The metacarpo-phalangeal joints were especially mobile in flexion, extension, abduction and adduction. The carpo-metacarpal joints were freely mobile. No other abnormalities were noted.

Radiographs (Fig. 4) showed that a triphalangeal thumb was present only on the right side. The middle phalanx on this side consisted of two wedge-shaped ossicles. The medial

VOL. 41 B, NO. 4, NOVEMBER 1959 
or ulnar ossicle was distinct and free, whereas the radial or lateral element appeared to be fused to the radial side of the head of the proximal phalanx. Both feet were radiographed and found to be normal.

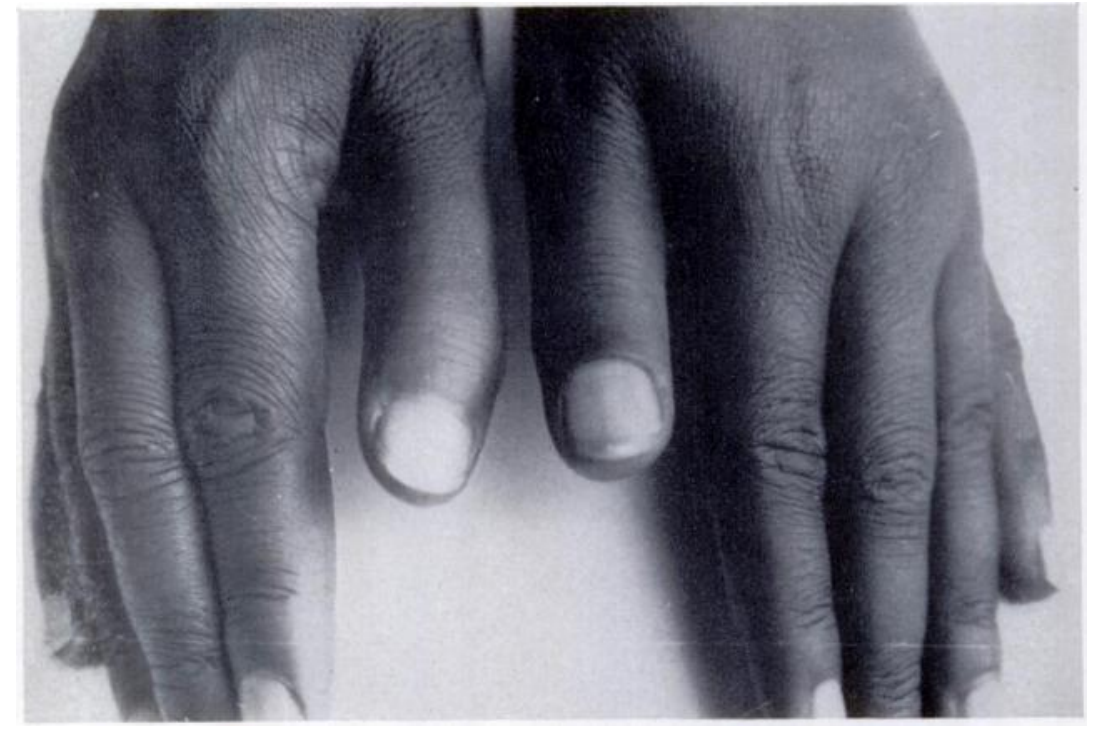

FiG. 3

Case 2

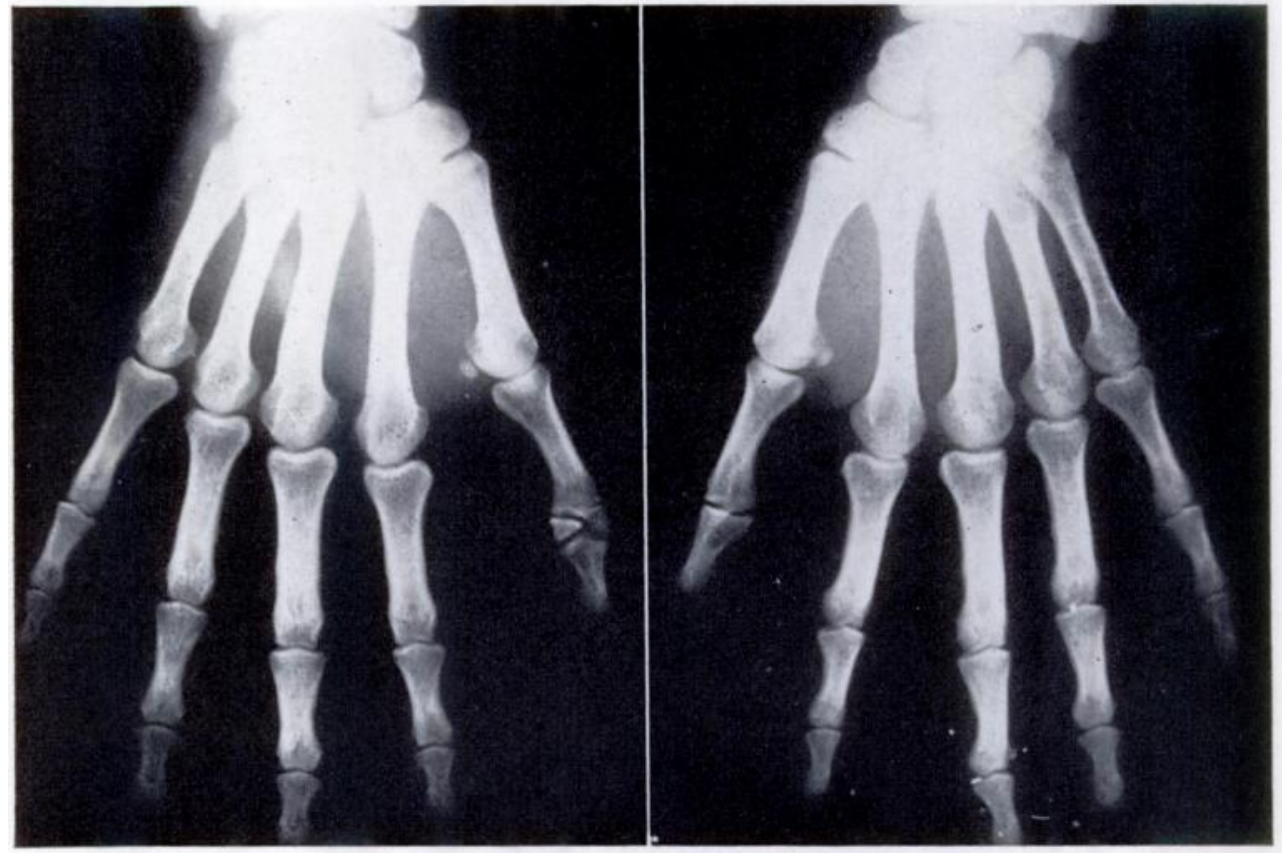

FIG. 4

Case 2

Case 3-The father of the woman in Case 2, a Bantu Shangaan man of sixty-seven years, was examined. Both his thumbs were relatively long, the tips of his thumbs reaching to the level of the middle of the middle phalanges of his index fingers (Fig. 5). Both terminal phalanges

THE JOURNAL OF BONE AND JOINT SURGERY 
were radially deviated. with restriction of movements at the distal interphalangeal joints. The dorsum of each thumb was smooth, with few creases. The metacarpo-phalangeal joints were freely mobile in all directions, as in Cases 1 and 2. No other anomalies were apparent.

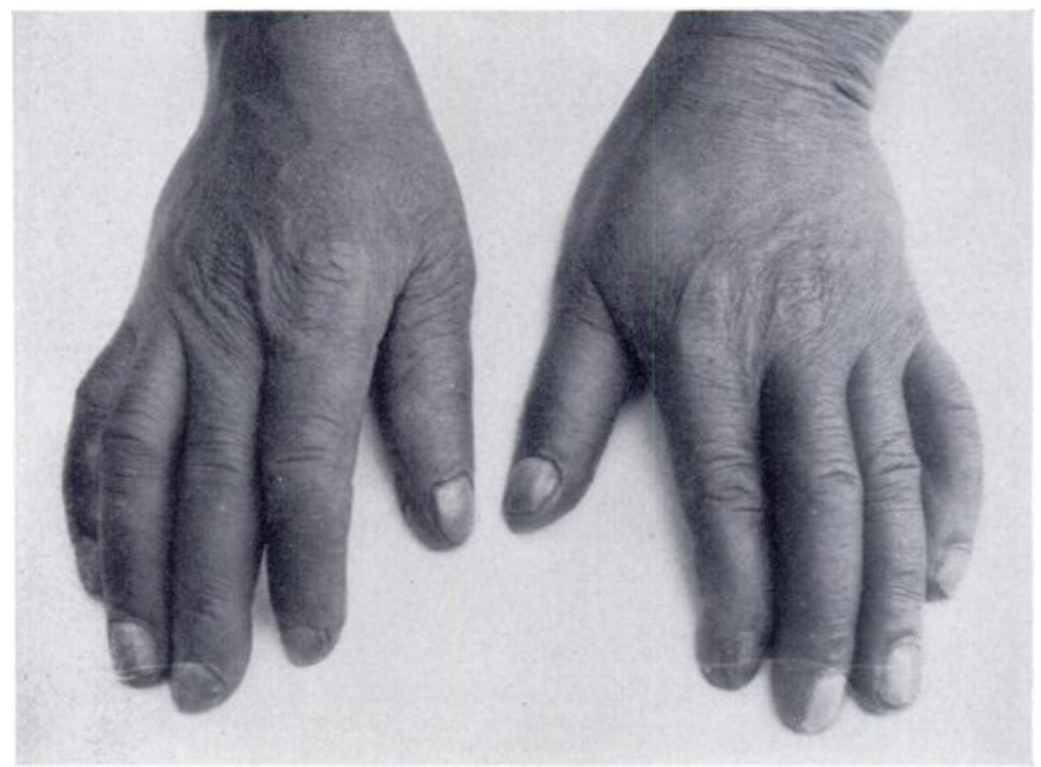

Fig. 5

Case 3

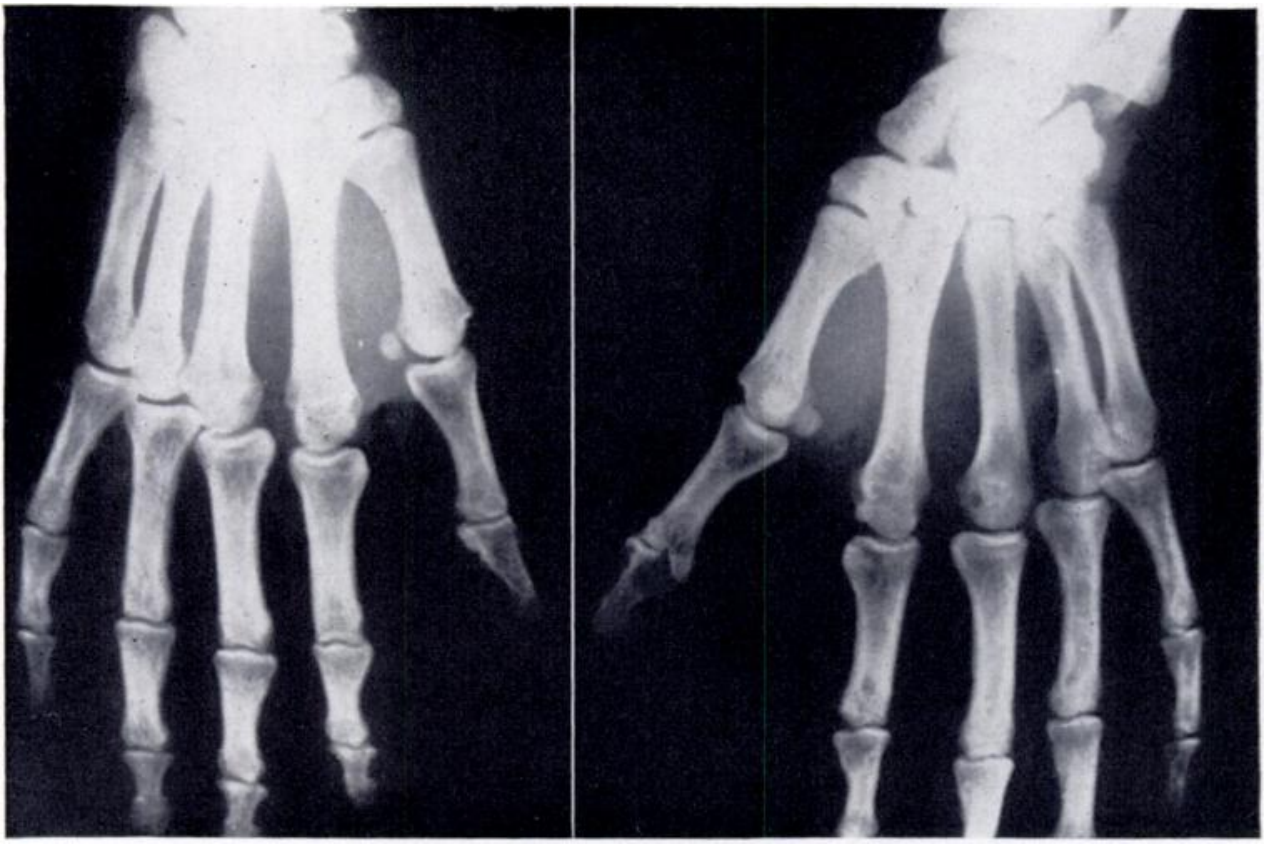

Fig. 6

Case 3

Radiographs of the hands (Fig. 6) did not show triphalangeal thumbs, but suggested that there might have been a triphalangeal thumb on the left. The distal end of the proximal phalanx presented a duckbill structure, in that two elements projected from the radial and 
ulnar sides respectively. The ulnar projection was the more prominent. It is suggested that these projections are comparable to the ossicles mentioned in Case 2, which had either not separated from, or had become fused with, the related proximal elements.

\section{DISCUSSION}

Etiology of triphalangeal thumb-Various hypotheses have been advanced to explain the presence normally of only two phalanges in the thumb. Galen (quoted by McGregor 1926) suggested that the thumb has no metacarpal, and that the so-called metacarpal is in fact a proximal phalanx. Those who favour this view point out that the thumb metacarpal usually has a basal epiphysis, and in this respect behaves more like the other phalanges. Moreover, in its structure and in its mobility restricted practically to one plane, the metacarpo-phalangeal joint of the thumb has the characteristics of an interphalangeal joint. The metacarpophalangeal joints of the other digits possess mobility for adduction and abduction movements as well as for flexion and extension. In all the cases described here there was free mobility at the metacarpo-phalangeal joints, suggesting that in these cases the joints behaved like those of the other digits.

There are, however, strong arguments against Galen's theory: thus a triphalangeal thumb may be associated, as exemplified in our series, with 1) capital epiphyses in the thumb metacarpals (Frazer 1940); and 2) both capital and basal epiphyses in the same thumb metacarpal (Gray's Anatomy 1949) (Fig. 2).

The evidence available suggests that the terminal phalanx of the normal thumb represents the fusion of two elements. This view was held by Frazer (loc. cit.), who drew attention to a

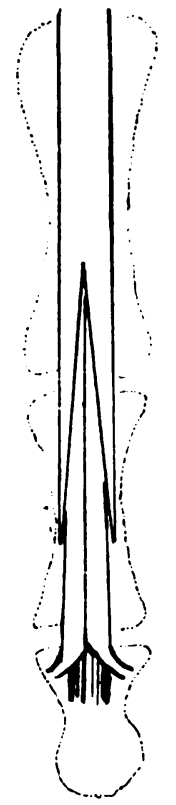

A

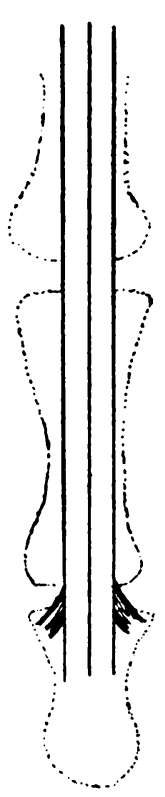

B

FIG. 7

A-Insertion of tendon of flexor digitorum profundus. B-Insertion of tendon of flexor pollicis longus. Note lateral fasciculi. similar occasional happening in the little toe of the foot. The fact that the terminal phalanx of the thumb in normal hands is longer than the distal phalanges of any of the other fingers, notwithstanding that the thumb is the shortest digit in the hand, is in my view an added argument in favour of this theory.

If this is acceptable, one must conclude that triphalangism in the human thumb represents the failure of fusion of the distal phalanx with a middle phalangeal element. Wilkinson (1953), who supported this view, dissected 100 fingers and thumbs and compared the nature of the insertions of flexor pollicis longus and its homologue flexor digitorum profundus. He showed that the superficial fibres of flexor digitorum profundus diverge from the midline ventral groove to be inserted on each side of the base of the ventral surface of the distal phalanx, exposing the deeper fibres which are inserted more distally (Fig. 7). In the case of the flexor pollicis longus the deeper fibres diverge, forming two distinct lateral fasciculi, which are inserted on each side of the ventral aspect of the base of the terminal phalanx of the thumb. The superficial fibres, however, pass vertically and distally to their attachment. Wilkinson believed that these fasciculi represent rudiments of a flexor sublimis tendon in the thumb, which has become fused with the profundus tendon. This, he suggested, has accompanied the fusion of the terminal and middle phalanges and caused the homologue of the sublimis tendon to become attached to that part of the fused element which corresponds to the middle phalanx. I have confirmed Wilkinson's observations, in the Bantu, in an earlier investigation (Abramowitz 1955). No account exists of a dissection of a triphalangeal thumb, 
and, as Wilkinson pointed out, "It would be interesting to see what the arrangement of the lateral fasciculi would be in a triphalangeal thumb."

Various other theories have been advanced regarding triphalangeal thumb. Joachimsthal (1900) believed that in some instances a triphalangeal thumb represents a duplication of the index finger with the absence of the thumb.

Haas (1939), in a study of three families, observed longitudinal splitting of the distal part of the nail phalanx, giving it a duckbill appearance. He asserted that triphalangeal thumb in its various forms represents arrested attempts at bifid thumb formation. The duckbilllike appearance of the middle phalanges in Cases 2 and 3 lend support to this theory.

The frequency with which triphalangeal thumb and bifid thumb are combined (Lapidus et al. 1943) stressed the close relationship of these two anomalies. Lapidus suggested that triphalangism of the thumb may be an incomplete development of the distal part of one of the two nail phalanges of a bifid thumb. If this is correct, the additional phalanx of a triphalangeal thumb is not a true middle phalanx, but a surviving portion of the base of one of the phalanges of a bifid thumb. The presence of ulnar deviation of the terminal phalanges of the thumbs, described in the present series, as well as by previous authors, suggests that the radial member in a bifid thumb was lost, while the ulnar member remained retaining its original ulnar position.

Hereditary aspects - Triphalangism of the thumb shows a definite hereditary pattern. Previous reports have drawn attention to this phenomenon, and in the present series there is little doubt that the anomalies are related in the three cases by direct hereditary influence. It is interesting that both the mother and her child (Cases 2 and 1 respectively) were first-born offspring.

\section{SUMMARY}

1. Three cases of triphalangeal thumb are described in three generations of a Bantu family. In the youngest member both thumbs were affected; in his mother and grandfather only one thumb was abnormal.

2. The literature is reviewed and the etiology of the anomaly is discussed.

3. Attention is drawn to the hereditary tendency of this condition.

I wish to express my appreciation to Professor D. J. du Plessis, head of the Department of Surgery, University of the Witwatersrand, for comments and suggestions in the preparation of this paper. I also wish to thank Mr B. Lewin, F.R.C.S., Senior Surgeon at the Coronation Hospital, in whose department these cases were seen, for helpful criticism and advice. My thanks are also due to Dr M. E. Davenport and Dr S. H. Getz of the Radiology Department, Coronation Hospital, for radiographs of the patients, and to Dr G. Elliot, Superintendent of the Coronation Hospital, for permission to publish this report.

\section{REFERENCES}

Abramowitz, 1. (1955): Anatomy of the Hand. Some Observations in the Bantu-speaking Negro. Leech, $25,31$. Frazer, J. E. (1940): The Anatomy of the Human Skeleton. Fourth edition, p. 118. London: J. \& A. Churchill. Frere, J. M. (1930): A Case Having Thumbs with Three Phalanges Simulating Fingers. Southern Medical Journal, 23, 536.

GALEN: quoted by McGregor, A. Lee (1926) loc. cit., 263.

Gray's Anatomy (1949): Thirtieth edition, p. 380. Edited by T. B. Johnston and J. Whillis. London: Longmans, Green \& Co.

HaAs, S. L. (1939): Three-Phalangeal Thumbs. American Journal of Roentgenology, 42, 677.

Joachimsthal (1900): Berliner Klinische Wochenschrift, 37, 835 (quoted by Lapidus, P. W.) loc. cit.

Lapidus, P. W., Guidotti, F. P., and ColettI, C. J. (1943): Triphalangeal Thumb. Surgery, Gynecology and Obstetrics, 77, 178.

McGregor, A. Lee (1926): A Contribution to the Morphology of the Thumb. Journal of Anatomy, 60, 259. Stieve, H. (1916): Úber Hyperphalangie des Daumens. Anatomischer Anzieger, 48, 565.

Struthers (1863): quoted by Sallam, A. M. (1955): Triphalangeal Thumbs. Archives of Surgery, 71, 257. Wilkinson, J. L. (1953): The Insertions of the flexores pollicis longus et digitorum profundus. Journal of Anatomy, 87, 75. 